\title{
Kampo medicines for gastrointestinal tract disorders: a review of basic science and clinical evidence and their future application
}

\author{
Kazunari Tominaga · Tetsuo Arakawa
}

Received: 18 February 2013/ Accepted: 20 February 2013/Published online: 16 March 2013

(C) Springer Japan 2013

\begin{abstract}
Treatment with kampo, the Japanese traditional medicine, is a form of pharmacological therapy that combines modern Western and traditional Asian medical practices. In Japan, various traditional medicines are often combined with Western medicines and prescribed for patients with diseases such as gastroesophageal reflux disease, functional dyspepsia, chronic gastritis, irritable bowel syndrome, and post-operative ileus. Based on numerous past observations, Japanese traditional medicines are thought to be particularly useful in the treatment of medically unexplained physical symptoms such as nausea, abdominal discomfort, and anorexia. However, the detailed mechanism by which they mediate their pharmacological action is yet unknown. In addition, the clinical evidence to support their use is insufficient. This review focuses on the basic evidence of the pharmacological action and the clinical efficacies of kampo medicines accumulated over several past decades. In addition, we introduce both the current novel insights into kampo medicines and the therapeutic approach employed when they are used to treat various disorders of the gastrointestinal tract.
\end{abstract}

Keywords Functional gastrointestinal disorders (FGIDs) . Gastroesophageal reflux disease (GERD) · Functional dyspepsia (FD) - Irritable bowel syndrome (IBS) .

Post-operative ileus · Traditional herbal medications . Gastrointestinal motility · Anti-inflammation

K. Tominaga $(\bowtie) \cdot T$. Arakawa

Department of Gastroenterology, Osaka City University

Graduate School of Medicine, 1-4-3, Asahimachi, Abeno-ku, Osaka 545-8585, Japan

e-mail: tomy@med.osaka-cu.ac.jp

\section{Introduction}

The Japanese traditional herbal medicine, kampo, is a form of pharmacological therapy that originated in medieval China and was developed further in Japan. Treatment with kampo means a possibility for combination therapy with modern Western and traditional Asian medical practices. Most such traditional medicines include various components, and they are often prescribed for patients with medically unexplained physical symptoms. However, the detailed mechanism(s) of the pharmacological action of kampo medicines is yet unknown. Consequently, a standardized educational system of these medicines has not been fully established until recently for medical students or trainees. In other words, the Japanese Ministry of Health and Welfare has approved the use of many kampo medicines as ethical medicines based on the symptoms of the respective patients. Therefore, therapeutic strategies that use kampo medicines based on proven evidence have yet to be established. Hence, kampo therapy is referred to as experience-based medicine and not evidencebased medicine, even though these medicines are prescribed in most clinical fields in Japan. However, extensive evidence for the clinical efficacies or pharmacological mechanism(s) of kampo medicines has gradually accumulated over past decades. This review focuses on the basic and clinical evidence for efficacies of kampo medicines in treating various disorders of the gastrointestinal tract (GI) tract and introduces the current novel insights in this field (Figs. 1, 2).

Gastroesophageal reflux disease (GERD) associated with esophageal motor function and visceral hypersensitivity

Recently, an increase in the prevalence of GERD has been reported in Japan and in Western countries owing to factors 
Fig. 1 Scheme of the pharmacological actions and functional site of kampo medicines for the upper GI tract

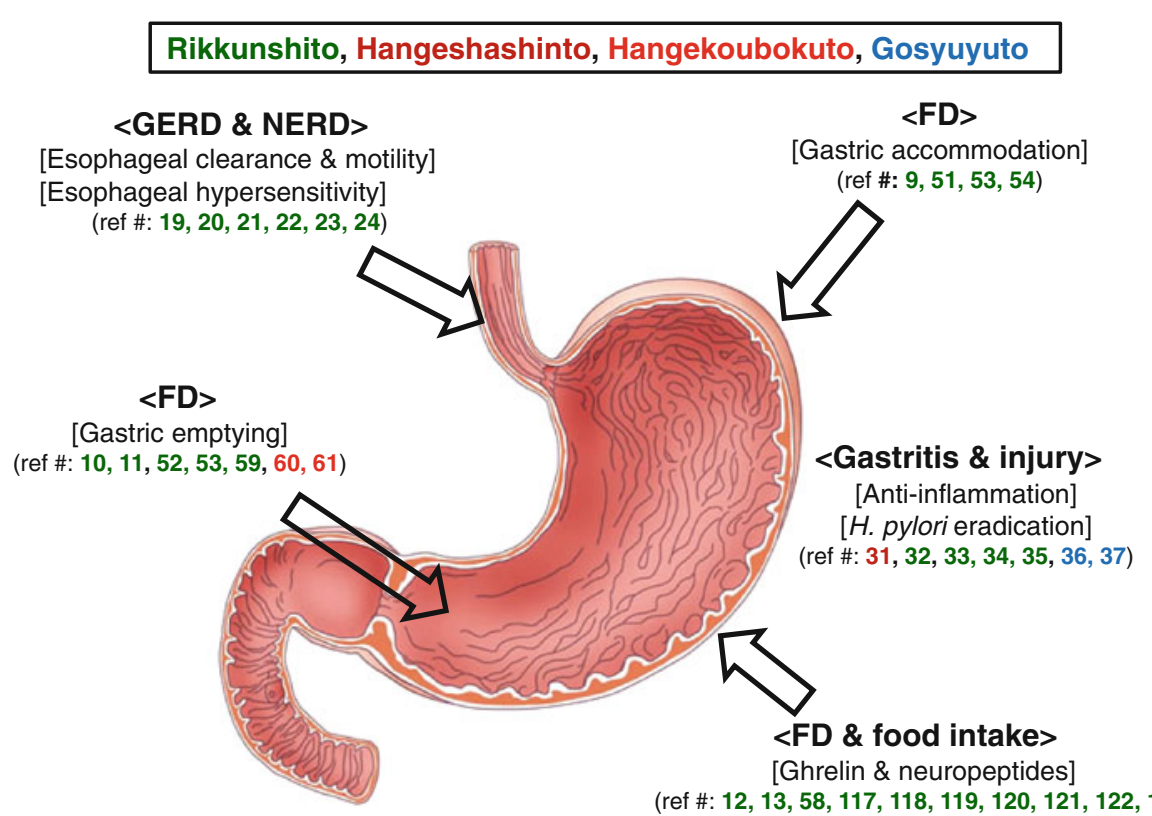

Rikkunshito, Hangeshashinto, Hangekoubokuto, Gosyuyuto

<GERD \& NERD> <FD>

Gastric accommodation

(ref \#: 9, 51, 53, 54)

(ref \#: 19, 20, 21, 22, 23, 24)

(ref \#: 12, 13, 58, 117, 118, 119, 120, 121, 122, 123 )

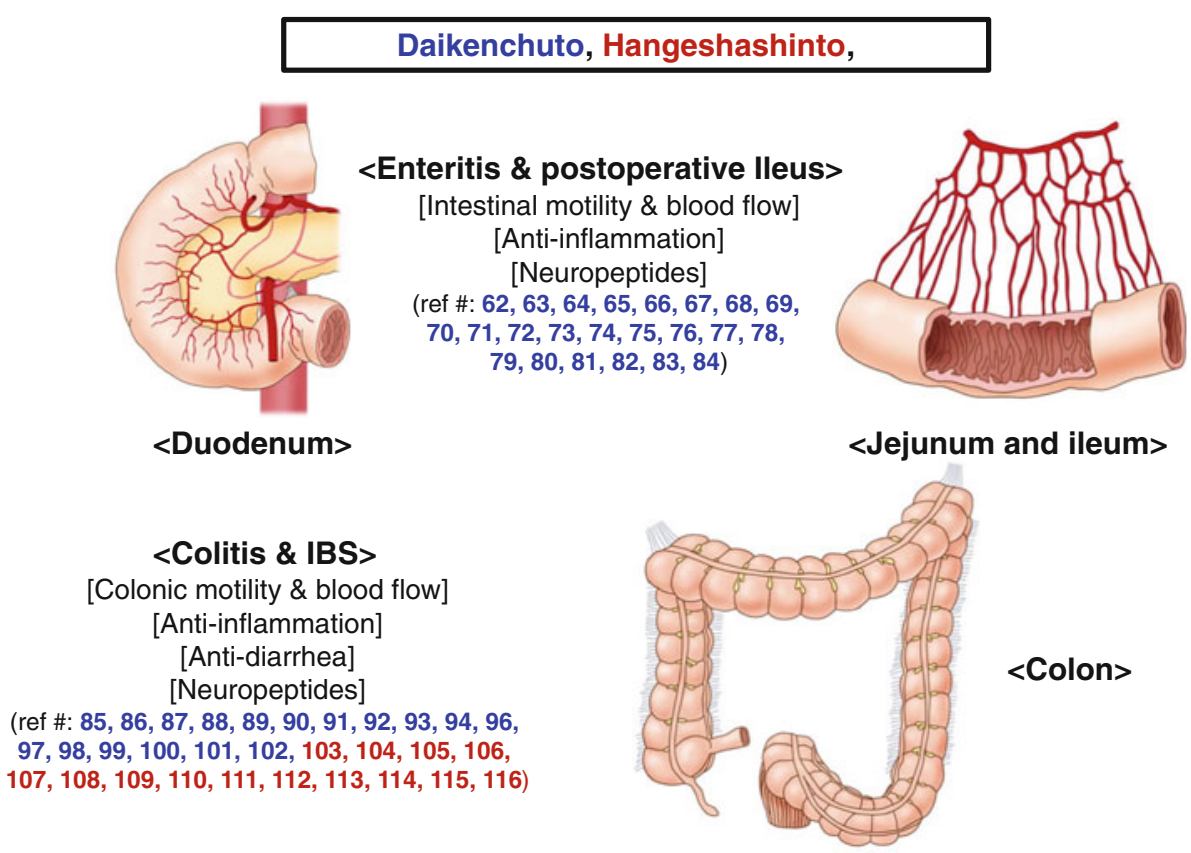

Fig. 2 Scheme of the pharmacological actions and functional sites of kampo medicines for the middle and lower GI tracts such as obesity associated with the intake of Western food, a decrease in the Helicobacter pylori infection rate, and increased prevalence of $H$. pylori eradication therapy [1-4]. The primary pathophysiology of GERD is the reflux of gastric contents, including gastric acid [5]. Esophageal motor dysfunctions such as an increase in transient lower esophageal sphincter relaxation (TLESR) are also associated with the occurrence of GERD [6]. Other factors contributing to the pathophysiology of GERD include esophageal peristalsis, mucosal hypersensitivity at the bottom of the esophagus to reflux contents such as gastric acid or bile acid, or disorders of gastric motility (gastric reservoir function or emptying). Hence, the inhibition of acid secretion by using proton pump inhibitors (PPIs) has been suggested as the most effective therapeutic strategy for GERD [7]. However, certain agents that regulate the physiological functions of the esophagus, e.g., its motor functions, may also prove useful in GERD treatment.

The Japanese traditional medicine rikkunshito has eight constituents, viz., Glycyrrhizae radix (4.7\%), Zingiberis rhizoma (2.3\%), Atractylodis lanceae rhizoma (18.6\%), Zizyphi fructus (9.3\%), Aurantii nobilis pericarpium (9.3\%), Ginseng radix (18.6\%), Pinelliae tuber (18.6\%), and Hoelen (18.6\%) [8], and is widely prescribed for 
patients with upper GI symptoms. Basic studies show that rikkunshito exhibits a pharmacological action similar to that of prokinetic agents, as shown by its attenuation of gastric dysmotility induced by a nitric oxide-synthesizing enzyme inhibitor $[9,10]$. In addition, rikkunshito improves the delay of gastric emptying mediated by serotonin (5-HT) type 3 receptor but not dopamine receptors [11]. Conversely, rikkunshito increases plasma ghrelin levels, and regulates motility at the esophagogastric junction [12, 13]. Ghrelin is known to have a strong orexigenic effect [14, 15] and enhances GI motility [16-18]. Nahata et al. [19] showed impairment of GI motility in a rat GERD model via dysfunction of ghrelin signaling and that rikkunshito restored GI motility by improving the ghrelin response. These data indicate the possible efficacy of rikkunshito in the treatment of GERD owing to its pharmacological action on motor functions throughout the upper GI tract.

Kawahara et al. [20] reported that rikkunshito improved nausea symptoms in children with GERD by increasing esophageal clearance and decreasing esophageal acid exposure time for the esophagus from a 24-h $\mathrm{pH}$ monitoring study. Rikkunshito is also effective for the relief of reflux symptoms such as heartburn and acid regurgitation [21]. Acid secretion inhibitors such as PPIs are the firstchoice agents for GERD treatment; however, we often encounter PPI-refractory GERD adult patients. Thus, antacid agents may not always be effective for GERD, especially in the case of a patient with no erosions on the esophageal mucosa (non-erosive reflux disease: [NERD]). In our recent report of a randomized, parallel comparative study of PPI-refractory GERD patients, we stated that rikkunshito combined with standard-dose rabeprazole therapy shows clinical efficacy similar to that of double-dose PPI [22]. In subgroup analyses of this study, the effectiveness of rikkunshito was more pronounced in male NERD patients, particularly leptosomatic NERD patients [22]. By using a rat reflux esophagitis model, Miwa et al. [23] showed that rikkunshito improves acid regurgitationassociated circumstances by promoting tight junction protein formation and suppressing the dilation of intercellular spaces in the epithelial mucosa. In addition to gastric acid reflux, bile acid reflux to the bottom part of the esophagus may also be an important factor in the pathogenesis of mucosal hypersensitivity leading to PPI-refractory GERD. Recently, Araki et al. [24] showed that rikkunshito exhibits potent and differential absorption of bile salts. Correctively, these findings suggest that rikkunshito may have the potential for improving hypersensitivity to gastric acid or bile acid at the bottom part of the esophagus. These pharmacological functions of rikkunshito different from motor function may suggest other explanations why it relieves the symptoms of PPI-refractory GERD patients, especially for NERD. Currently, double-dose PPI and long-term maintenance therapy is the standard therapeutic strategy for such PPI-refractory GERD patients [25]; however, a new regimen that includes rikkunshito should be considered for GERD patients including PPI-refractory cases.

\section{Mucosal injuries and histological gastritis (H. pylori-induced gastritis)}

Acute inflammation of the stomach is often caused by factors such as non-steroidal anti-inflammatory drugs (NSAIDs), ethanol, systemic stress, or H. pylori infection, all of which may cause mucosal injuries [26-28]. Acid secretion inhibitors, such as PPIs and histamine type 2 receptor antagonists (H2RAs), or mucoprotective agents, such as rebamipide and teprenone, are effective for treating mucosal injuries [29, 30]. Aside from these drugs, some traditional medicines are also used to protect against acute gastric injuries. Kase et al. [31] reported that hangeshashinto and shosaikoto inhibited ethanol-induced gastric mucosal injuries in a dose-dependent manner by eliminating oxygen radicals. Hangeshashinto is also reported to elevate secretion of mucin, which protects against acute gastric mucosal injuries. Hangeshashinto improves mucosal injuries caused by water-immersion stress and an acute mucosal injury model; however, shosaikoto did not show this property. This disparity in their effects may be due to differences in the respective components of these medicines. Furthermore, rikkunshito exerts a protective effect against gastric mucosal injuries induced by ethanol. The effect of rikkunshito is mediated by the inhibition of decrease in mucin content of the gastric mucosa [32], and this effect is in part mediated by nitric oxide (NO) but not prostaglandins or sulfhydryls [33]. Thus, these findings suggest that both hangeshashinto and rikkunshito exert protective effects against acute mucosal injuries in the stomach of rats.

Chronic gastritis, which is characterized by chronic infiltration of inflammatory cells, hypertrophy or atrophy of the gastric glands, or intestinal metaplasia, has been proved to be caused by persistent $H$. pylori infection. Therefore, chronic gastritis is also referred to as histological gastritis, the standard treatment for which includes triple antimicrobial therapy. However, some traditional herbal medicines have shown evidence of antimicrobial efficacy against $H$. pylori. Ishii reported that 104 of 109 kinds of medicinal plants and 28 types of spices had anti-H. pylori activities [34]. Among all crude drugs, gosyuyu had a markedly higher antibacterial activity against $H$. pylori in vitro. Gosyuyuto has four constituents; Evodia fructus, Zizyphi fructus, Zingiberis rhizoma, and Ginseng radix. In a clinical study report, Higuchi et al. [35] reported that 
gosyuyuto combined with omeprazole and amoxicillin yields a higher eradication rate $(84 \%)$ than dual therapy $(59 \%)$ without causing any significant adverse effect. Moreover, Hamasaki et al. [36] analyzed the components of gosyuyuto and reported that two quinolone compounds (AM quinolones), 1-methyl-2-[(Z)-8-tridecenyl]-4-(1H)quinolone and 1-methyl-2-[(Z)-7-tridecenyl]-4-(1H)-quinolone, which were purified from the Evodia fructus have strong and selective antibacterial activity against the reference strains and isolated strains of $H$. pylori from clinical patients. Further, in vitro studies revealed that the antibacterial effect of these two AM quinolones against H. pylori is mediated by the inhibition of respiration and not DNA synthesis, and in vivo studies revealed that they significantly decreased the number of viable $H$. pylori in the stomachs of $H$. pylori-infected Mongolian gerbils and reduced neutrophil infiltration without causing harmful adverse events, including animal mortality [37]. The agents with a unique antimicrobial mechanism(s) mediated by the inhibition of bacterial respiration, i.e., mechanisms different from that of antibiotics such as amoxicillin, clarithromycin, or metronidazole, may be beneficial for the improvement of $H$. pylori-associated gastric diseases, whether they are used alone or in combination with the above mentioned antibiotics and PPIs. Thus, there are some candidates among traditional medicines to treat or protect against gastric mucosal injuries caused by the above various mechanism(s), including $H$. pylori infection.

\section{Functional dyspepsia (FD)}

Another form of chronic gastritis in Japan is a symptomatic gastritis called functional dyspepsia (FD), and it is classified as a functional GI disorder (FGID). According to the Rome III criteria published in 2006 [38], the diagnosis of FGIDs is based on the following conditions: patients exhibit symptoms originating from the GI tract without evident organic disease, and these bothersome symptoms persist even after various treatments, thus decreasing their quality of life (QOL) [39]. FD has four representative symptoms, viz., epigastric pain, epigastric burning, postprandial fullness, or early satiety. It is common worldwide, and its prevalence has increased recently. The pathophysiology of FD is characterized by multifunctional disorders of the upper GI tract, e.g., disorders of GI motility [40], abnormal acid secretion [41], visceral hypersensitivity [42], H. pylori infection [43], psychological factors [44, 45], and imbalance of the autonomic nervous system (ANS) [46]. Of these factors, most studies mainly focus on the disorders of GI motility, because they are closely related to the pathogenesis of FD. In general, gastric motility consists of three phases: reservoir, churning, and emptying. While previous reports have mainly focused on the delay in gastric emptying [47], it is necessary to consider the total coordination of these phases for the better understanding of FD. Gastric accommodation, a reservoir function, originates from the proximal stomach and is important for the physiological function of the stomach. Neurological transmitters such as NO that are essential for this function have already been elucidated [48, 49]. Impairment of gastric accommodation has also been reported to cause clinical symptoms such as epigastric discomfort, early satiety, and bloating of FD patients [50]. Furthermore, we have previously reported that the impairment of reservoir function is associated with delay in gastric emptying, which results in dyspeptic symptoms [40]. Thus, drugs that can improve the impairment of reservoir function of the stomach are desirable. With regard to traditional medicines, the kampo medicine rikkunshito regulates GI motility in a manner similar to prokinetic drugs. Hayakawa et al. [9] reported that, in isolated guinea pig stomachs, rikkunshito promotes adaptive relaxation, which means gastric accommodation. Rikkunshito also induces the relaxation of gastric fundus smooth muscles isolated from diabetic neuropathic rats with gastric dysmotility [51]. In addition, rikkunshito can improve gastric dysmotility mediated by NO or 5-HT3 receptor pathways $[10,11]$. Considering this basic evidence, rikkunshito may be a promising drug for the treatment of FD in the clinical field.

Unfortunately, an appropriate treatment regimen for FD has not yet been established, even though a variety of clinical trials considering the above pathogenesis have been conducted in Japan. Therefore, treatment may be empirically adopted for FD patients based on the predominantly suspected pathogenesis related to respective symptoms of FD. There is some evidence for the effectiveness of kampo medicines in the treatment of FD under such conditions. When FD was still called non-ulcer dyspepsia (NUD), Tatsuta et al. [52] illustrated the efficacy of rikkunshito on gastric emptying in patients with NUD, which supports the data shown in the above basic studies. Interestingly, in addition to this effect on gastric motility, rikkunshito also improved GI symptoms in patients with NUD [52]. To our knowledge, this is the first report in the English literature that describes rikkunshito as a promising drug for the improvement of symptoms of FD patients. Kusunoki et al. [53] showed that rikkunshito may be beneficial for the treatment of FD patients with impaired gastric accommodation and gastric motility evaluated by the extracorporeal ultrasonography method. On the other hand, physical and psychological stress often causes gastric hypersensitivity to stimulation by mechanical balloon distension, which is generally well known as a pathogenesis of FD. Shiratori et al. [54] showed that rikkunshito may 
improve stress-induced gastric hypersensitivity and/or changes in gastric wall tone by using gastric barostat method. One report assessed the efficacy of some herbal medicines under the condition of venipuncture stress. Rikkunshito and hangeshashinto but not hangekobokuto or ninjinto reversed the increase in plasma levels of neuropeptide $\mathrm{Y}$, a representative neurotransmitter of the sympathetic nervous system of ANS, to the control levels [55]. In addition, administration of rikkunshito decreases the afferent activity of the gastric vagal nerve, but increased the efferent activities of the gastric branches of the vagal nerve [56]. From these findings with the fact that activity of sympathetic nervous system in the ANS of FD patient was relatively higher compared to that of the parasympathetic nervous system [57], the above might be another pharmacological action of rikkunshito for the improvement of dyspeptic symptoms. According to another comparative human study, rikkunshito but not domperidone also improves symptoms of patients with FD in parallel with plasma ghrelin levels [58]. Rikkunshito also has an impact on delayed gastric emptying in severely handicapped patients [59] and on the coordination of the gastric myoelectric activity in post-operative dyspeptic children after GI surgery [60]. These findings suggest that rikkunshito improves dyspeptic symptoms mediated by regulating gastric sensorimotor function or some inflammatory and/or neuroendocrinal mediators. Oikawa et al. also reported the efficacy of hangekoubokuto in FD patients. This efficacy is mediated by a reduction of gas volume in parallel with the improvement of symptoms of abdominal pain, indigestion, and constipation [61]. Thus, there are some candidates among various Japanese traditional medicines for the treatment of both histological and symptomatic gastritis.

\section{Motility disorders and inflammations of the small intestine}

The physiological function of the small intestine mainly comprises ingestion and absorption of foods throughout GI transit. Spontaneous peristaltic contraction is vital for the performance of these functions. Therefore, a regulated intestinal motility is important to maintain its integrity and achieve these functions. However, disorders of intestinal motility often occur under some pathological conditions due to surgical operation and abdominal irradiation for treating some neoplasms, the unfavorable conditions of which lead to serious symptoms. Therefore, some effective drugs including Japanese traditional medicines that regulate intestinal motility are needed in the clinical field. Apart from rikkunshito, a variety of reports have documented the effectiveness of daikenchuto in such cases. Daikenchuto is composed of Zanthoxyli fructus, Ginseng radix, Zingiberis siccatum rhizoma, and maltose syrup powder and generally accelerates intestinal motility in various animal models of intestinal dysmotility, viz., mouse [62, 63], rat [64-66], guinea pig [67, 68], rabbit [69], or dog [70, 71]. The pharmacological functions of this drug are usually mediated by an increase in acetylcholine release through the vagal nerve, cholinergic neuron, or 5-HT3 and/or 5-HT4 receptors [64-67, 70-72]. Another report showed that a possible role of daikenchuto in postoperative adhesive obstruction via transient receptor potential vanilloid type 1 channel [73]. In addition, daikenchuto exhibit anti-inflammatory activity in rat intestinal mucosal injuries, mediated by decreased expressions of interleukin-1 and interferon (IFN)- $\gamma$ [74] and in bacterial translocation models, mediated by reducing inflammatory reaction and maintaining intestinal integrity [75]. Further examinations showed that the main component exerting this pharmacological function is hydroxy sanshool, one of the chief constituents of Zanthosyli fructus included in daikenchuto [66, 69-71, 76]. These findings from various basic studies correctively suggest that daikenchuto may be useful for the treatment of post-operative adhesive obstructions or ileus.

In clinical studies, a similar action of daikenchuto on intestinal motility has been reported. Manabe et al. [77] showed that daikenchuto led to an increase in intestinal motility in a randomized placebo controlled study in healthy humans. Another study with human subjects suggested that the effect of daikenchuto on intestinal transit may be mediated by the releases of motilin and vasoactive intestinal peptide (VIP) into the plasma through muscarinic receptor 1 [78]. Daikenchuto is reported to improve the stasis of patients with total gastrectomy and jejunal pouch interposition [79], decrease the prevalence rate of re-operation associated with ileus after abdominal surgery [80], and attenuate radiation-induced enteritis [81]. Thus, it was concluded that the pharmacological action of this drug is mainly mediated by improvement of intestinal motility. Interestingly, daikenchuto is also reported to increase human portal blood flow mediated by an increase in the intestinal blood flow without an increase in the cardiac output $[82,83]$. It is also confirmed that an increase in intestinal blood flow by daikenchuto is mainly mediated by calcitonin gene-related peptide (CGRP) [84]. Thus, Japanese traditional medicines could regulate the physiological function of the small intestine, particularly, intestinal motility, although definitive evidence is limited only to daikenchuto at present.

\section{Colonic motility, secretions, and inflammation-colitis and irritable bowel syndrome}

Colonic transit in cooperation with mucus secretions plays an important role in the regulation of fecal conditions, such 
as constipation and diarrhea, after ingestion of food intake. Constipation and diarrhea are usually recognized as the common complaints but are not associated with severe lifethreatening diseases in the general clinical field. It is also well known that there seems to be a high prevalence of patients with these symptoms, including those individual who have never visited any medical institutions despite having these unfavorable conditions. In addition to the efficacy of Japanese traditional medicines for intestinal contraction, some of these medicines can also regulate colonic motility and improve fecal conditions and their related symptoms. In basic studies, daikenchuto has been found to exhibit prokinetic effects on the total GI tract, including the stomach, duodenum, jejunum, ileum, and colon during the fasting state [85]. However, this effect was attenuated during the fed state when daikenchuto was administered orally. Conversely, intracolonic administration of daikenchuto induced a colonic contraction similar to that during both the fasting and the fed states, and the related defecation occurred despite the differences in administration site and timing [85]. Based on these findings, the focal administration of this drug may be optimal for the treatment of intestinal obstruction, such as postoperative ileus. Moreover, daikenchuto improves the opioidinduced disorders of the colonic motility, which is similar to that seen by naloxone. As a result, Wood et al. [86] concluded that daikenchuto may have a beneficial effect on the restoration of colonic motility in postoperative patients with opiate analgesic therapy. Human studies revealed that the prokinetic effect of daikenchuto on colonic motility was mediated by increase in the levels of plasma motilin, VIP, and 5-HT [87, 88]. On the other hand, Kono et al. [89] showed that, in rats, daikenchuto can also increase blood flow in the colon via CGRP and receptor-activity modifying protein 1. In another human study, increase in the blood flow by daikenchuto was mediated by increase in the levels of plasma CGRP and substance P [90].

In a clinical study with healthy volunteers, occurrence of colonic motility was immediately confirmed by extrasomatic ultrasonography for $30 \mathrm{~min}$ after administration of daikenchuto into the ascending colon. However, no colonic contractions were observed in the right colon after administration of saline solution alone [91]. In patients hepatectomized by laparotomy, paralytic ileus occasionally occurs as a complication during the early postoperative period, which often causes post-resection liver failure due to hyperammonemia. Daikenchuto can prevent such unfavorable condition by improvement of delayed flatulence and hyperammonemia [92], and also accelerate the times to initial flatus and tolerance of regular diet by increasing bowel movement in patients after colorectal surgery [93]. Thereafter, it has been confirmed that daikenchuto improves some disorders of the colon such as obstructive bowel disease [94] or anorectal dysfunction with severe constipation in children [95, 96], and constipation in patients with Parkinson's disease [97]. Based on these findings, daikenchuto has been recognized as a prokinetic agent that is effective in the treatment of constipation and postoperative motility disorders.

Furthermore, daikenchuto exhibits anti-inflammatory effects. In a representative experimental colitis model induced by trinitrobenzene sulfonic acid (TNBS), daikenchuto ameliorated microvascular dysfunction via the regulation of endogenous adrenomedullin [98]. Furthermore, daikenchuto inhibits mucosal injuries and adhesion of the colonic serosa caused by focal inflammation and decreases cytokine levels of tumor necrosis factor and IFN$\gamma$ via the same mechanism [99]. As the pathophysiology of irritable bowel syndrome (IBS), low grade inflammation of the intestine is associated with its hypercontractility leading to clinical diarrhea. Therefore, both regulation of colonic motility and inflammation are important for the therapeutic strategy. Intestinal muscle hypercontractility associated with inflammation in a T cell-mediated model of enteropathy was ameliorated by daikenchuto, which indicated that this pharmacological function was mediated by decrease in pro- and anti-inflammatory cytokines [100]. Therefore, the possibility of daikenchuto for treating patients with IBS was suggested. As another function of daikenchuto, Inoue et al. [101] reported the regulation of intestinal fibrosis associated with decreasing expression of heat shock protein 47 and collagen content in this model. Radiation-induced enteritis occurs during radiotherapy for pelvic cancers. This disease often impairs the QOL of the affected individual. Unfortunately, there is no definite therapeutic rationale for this disease. However, one case report states that daikenchuto has the potential to manage radiation-induced enteritis perhaps owing to its antiinflammatory action [81]. In a retrospective observational clinical study, daikenchuto reduced the duration of longtube decompression and saved costs by prevention of postoperative adhesive small bowel obstruction, which is a major complication of abdominal surgery [102]. The comparative study showed that daikenchuto lowered the inflammatory responses associated with laparoscopic colorectal resection and shortened the time to first flatus [103]. Thus, daikenchuto may be useful as a prokinetic and anti-inflammatory drug under various pathological conditions.

Further, hangeshashinto has a potent and special effect on the lower intestinal tract. Through a series of pharmacological studies of hangeshashinto for colonic functions, Kase et al. have accumulated several basic evidences regarding its anti-diarrheal effects. Firstly, they showed the dose-dependent effects of this drug via an increase in water absorption in the colon but not inhibition of colonic transit 
in a castor oil-induced diarrhea model [104, 105]. The other mechanism(s) of anti-diarrheal effects of hangeshashinto was proved by increases in serum corticosterone levels and fluid secretion. It was also confirmed that a decrease in prostaglandin E2 contents associated with antidiarrheal effects was caused by an inhibition of cyclooxygenase type 2 . In addition, there are some reports on the association between hangeshashinto and GI motility regulatory peptides (somatostatin, motilin, gastrin, and VIP) or neuropeptides (5-HT, CGRP, and substance P). Hangeshashinto increases the plasma levels of somatostatin, motilin, gastrin, CGRP, and substance $\mathrm{P}$, but did not have an effect on the levels of 5-HT, mineral balance, or aldosterone [105-110]. Hangeshashinto also improved macroscopic injuries and body weight loss in a TNBS-induced colitis model [111]. Unfortunately, thus far, pharmaceutical evidence for hangeshashinto might be derived only from these complicated interactions. However, hangeshashinto also has the potential to maintain the integrity of the GI tract, including the improvement of clinical colitis.

\section{Miscellaneous: supportive care during systemic chemotherapy}

In the clinical field, most clinicians often encounter various patients with general malaise and have trouble treating these patients in coordination with the severity of their symptoms. General malaise includes anorexia, headache, dizziness, nausea, emesis, discomfort, and diarrhea. Furthermore, because the QOL of these patients lowers drastically with the severity of their malaise, clinicians need to improve their symptoms as soon as possible. In such cases, kampo medicines are used more frequently than the usual common drugs as comprehensively alternative medicines, because it is well known that kampo medicines can affect the physiological as well as the mental conditions. Therefore, kampo therapy may be the logically correct strategy for treatment in patients with numerous general malaise. However, it is difficult to establish the basic and clinical evidence for kampo medicines in the treatment of general malaise. Among various pathological conditions, it is well known that systemic chemotherapy using anti-cancer drugs causes symptoms similar to general malaise including anorexia, emesis, or diarrhea, which sometimes lead to serious life-threatening conditions. For instance, irinotecan hydrochloride (CPT-11) is often effectively used to treat cancers of the lung, colon, or ovarian cancers but can cause severe diarrhea as an adverse event. There is some evidence that hangeshashinto helps alleviate such adverse events. Hangeshashinto prevented CPT-11-induced symptoms such as diarrhea, anorexia, and body weight loss in rats, without a loss of its antitumor effects $[112,113]$. In the same model, hangeshashinto led to an increase in water absorption in the colon, mediated by an inhibition of prostaglandin E2 synthesis [114]. Another clinical study confirmed the preventive effect of hangeshashinto toward CPT-11-induced diarrhea in patients with advanced nonsmall-cell lung cancer [115]. We believe that, in the current literature, there is some evidence supporting the efficacy of this agent in patients with gastric or colon cancers.

Apart from diarrhea, anorexia is another critical cause for discontinuation of chemotherapy. Therefore, a candidate drug improving anorexia may be useful in such cases. However, only a limited number of agents that can effectively increase food intake have been established. Some reports show that rikkunshito can regulate some humoral factors, including gut regulatory peptides (somatostatin, gastrin, VIP, or CGRP) [110, 116], and improve stressassociated diseases by decreasing the levels of adrenocorticotropic hormone or cortisol [117]. Considering these effects of rikkunshito and its efficacy in patients with FGIDs, rikkunshito could serve as candidate supporting drug for improving systemic general malaise. Recently, it has been proved that rikkunshito can increase food intake and improve anorexia under unfavorable condition by increasing in plasma ghrelin levels. First, Takeda et al. [12] showed that rikkunshito suppresses cisplatin-induced anorexia in rats via 5-HT2 receptor antagonism. Thereafter, Yakabi et al. [13, 118] showed that rikkunshito led to improvement for the reduced secretion of ghrelin in the hypothalamus and resulted in the improvement of cisplatininduced anorexia. Another report states that a component of rikkunshito (10-Gingerol) improves cisplatin-induced anorexia by inhibiting acylated ghrelin degradation [119]. Basic research shows that rikkunshito suppresses anorexia, emesis, or decrease in QOL under the condition of systemic chemotherapy with cisplatin etc. by increasing the secretion of plasma ghrelin $[120,121]$. Thus, rikkunshito may be useful for supportive care during chemotherapy. Therefore, some kampo medicines seem to be more and more useful for treatment for general malaises in the future.

\section{Concluding remarks}

The Japanese medical treatment kampo is frequently prescribed for patients with numerous GI tract disorders associated with various symptoms, including general malaise. This treatment should be recommended theoretically, because it combines modern Western and traditional Asian medical practices. However, kampo medicines are not used widely worldwide and are considered "complementary" medicines and not "mainstream". This is because the mechanism of their pharmacological actions is not so clear and because of the lack of established kampo educational 
system in Japan. It is suggested that kampo medicines must be developed to the evidenced-based medicine from the empirical-based medicine in the near future by making known their much more benefits to the whole world.

Conflict of Interest Kazunari Tominaga received a research grant from a Grant-in-Aid for Scientific Research from the Ministry of Education, Culture, Sports, Science, and Technology of Japan. Tetsuo Arakawa received a research grant from a Grant-in-Aid for Scientific Research from the Ministry of Education, Culture, Sports, Science, and Technology of Japan; received lecture fees from Eisai Co. Ltd.; received financial support Otsuka pharmaceutical Co. Ltd, Astellas pharmaceutical Co. Ltd, Abbott Japan Co. Ltd, Eisai Co. Ltd, DaiichiSankyo Co. Ltd, Dainipponsumitomo pharmaceutical Co. Ltd, Takeda pharmaceutical Co. Ltd.

\section{References}

1. Furukawa N, Iwakiri R, Koyama T, Okamoto K, Yoshida T, Kashiwagi Y, et al. Proportion of reflux esophagitis in 6010 Japanese adults: prospective evaluation by endoscopy. J Gastroenterol. 1999;34:441-4.

2. Shimazu T, Matsui T, Furukawa K, Oshige K, Mitsuyasu T, Kiyomizu A, et al. A prospective study of the prevalence of gastroesophageal reflux disease and confounding factors. J Gastroenterol. 2005;40:866-72.

3. Mishima I, Adachi K, Arima N, Amano K, Takashima T, Moritani M, et al. Prevalence of endoscopically negative and positive gastroesophageal reflux disease in the Japanese. Scand J Gastroenterol. 2005;40:1005-9.

4. Fujiwara Y, Arakawa T. Epidemiology and clinical characteristics of GERD in the Japanese population. J Gastroenterol. 2009;44:518-34.

5. Vakil N, van Zanten SV, Kahrialis P, Dent J, Jones R. the Global Consensus Group. The Montreal definition and classification of gastroesophageal reflux disease: a global evidence-based consensus. Am J Gastroenterol. 2006;101:1900-20.

6. Dodds WJ, Hogan WJ, Helm JF, Dent J. Pathogenesis of reflux esophagitis. Gastroenterology. 1981;81:376-94.

7. Coté GA, Howden CW. Potential adverse effects of proton pump inhibitors. Curr Gastroenterol Rep. 2008;10:208-14.

8. Hattori T, Fujitsuka N, Asakawa A, Inui A. A strategy using Rikkunshito (Liu-Jun-Zi-Tang), a Japanese traditional medicine, to treat gastrointestinal disease. In: Basics of evidencesbased herbal medicine. Kerala: Research Signpost Press; 2010. p. 149-60.

9. Hayakawa T, Arakawa T, Kase Y, Akiyama S, Ishige A, Takeda $\mathrm{S}$, et al. Liu-Jun-Zi-Tang, a kampo medicine, promotes adaptive relaxation in isolated guinea pig stomachs. Drugs Exp Clin Res. 1999;25:211-8.

10. Kido T, Nakai Y, Kase Y, Sakakibara I, Nomura M, Takeda S, et al. Effects of rikkunshi-to, a traditional Japanese medicine, on the delay of gastric emptying induced by N(G)-nitro-L-arginine. J Pharmacol Sci. 2005;98:161-7.

11. Tominaga K, Kido T, Ochi M, Sadakane C, Mase A, Okazaki H, et al. The traditional Japanese medicine rikkunshito promotes gastric emptying via the antagonistic action of the 5-HT3 receptor pathway in rats. Evid Based Complement Alternat Med. 2009;1-8. doi:10.1093/ecam/nep173.

12. Takeda H, Sadakane C, Hattori T, Katsurada T, Ohkawara T, Nagai K, et al. Rikkunshito, an herbal medicine, suppresses cisplatin-induced anorexia in rats via 5-HT2 receptor antagonism. Gastroenterology. 2008;134:2004-13.
13. Yakabi K, Sadakane C, Noguchi M, Ohno S, Ro S, Chinen K, et al. Reduced ghrelin secretion in the hypothalamus of rats due to cisplatin-induced anorexia. Endocrinology. 2010;151: 3773-82.

14. Kojima M, Hosoda $\mathrm{H}$, Date $\mathrm{Y}$, Nakazato M, Matsuo $\mathrm{H}$, Kangawa K. Ghrelin is a growth-hormone-releasing acylated peptide from stomach. Nature. 1999;402:656-60.

15. Nakazato M, Murakami N, Date Y, Kojima M, Matsuo H, Kangawa K, et al. A role for ghrelin in the central regulation of feeding. Nature. 2001;409:194-8.

16. Fujino K, Inui A, Asakawa A, Kihara N, Fujimura M, Fujimiya M. Ghrelin induces fasted motor activity of the gastrointestinal tract in conscious fed rats. J Physiol. 2003;550:227-40.

17. Inui A, Asakawa A, Bowers CY, Mantovani G, Laviano A, Meguid MM, et al. Ghrelin, appetite, and gastric motility: the emerging role of the stomach as an endocrine organ. FASEB J. 2004;18:439-56.

18. Masuda Y, Tanaka T, Inomata N, Ohnuma N, Tanaka S, Itoh Z, et al. Ghrelin stimulates gastric acid secretion and motility in rats. Biochem Biophys Res Commun. 2000;276:905-8.

19. Nahata M, Muto S, Oridate N, Ohnishi S, Nakagawa K, Sadakane $\mathrm{C}$, et al. Impaired ghrelin signaling is associated with gastrointestinal dysmotility in rats with gastroesophageal reflux disease. Am J Physiol Gastrointest Liver Physiol. 2012;303: G42-53.

20. Kawahara H, Kubota A, Hasegawa T, Okuyama H, Ueno T, Ida $\mathrm{S}$, et al. Effects of rikkunshito on the clinical symptoms and esophageal acid exposure in children with symptomatic gastroesophageal reflux. Pediatr Surg Int. 2007;23:1001-5.

21. Hiyama T, Yoshihara M, Tanaka S, Haruma K, Chayama K. Strategy for treatment of nonerosive reflux disease in Asia. World J Gastroenterol. 2008;14:3123-8.

22. Tominaga K, Iwakiri R, Fujimoto K, Fujiwara Y, Tanaka M, Shimoyama Y, et al. Rikkunshito improves symptoms in PPIrefractory GERD patients: a prospective, randomized, multicenter trial in Japan. J Gastroenterol. 2012;47(3):284-92.

23. Miwa H, Koseki J, Oshima T, Kondo T, Tomita T, Watari J, et al. Rikkunshito, a traditional Japanese medicine, may relieve abdominal symptoms in rats with experimental esophagitis by improving the barrier function of epithelial cells in esophageal mucosa. J Gastroenterol. 2010;45:478-87.

24. Araki Y, Mukaisho KI, Fujiyama Y, Hattori T, Sugihara H. The herbal medicine rikkunshito exhibits strong and differential adsorption properties for bile salts. Exp Ther Med. 2012;3(4): 645-9.

25. Kinoshita Y, Hongo M. Japan TWICE Study Group. Efficacy of twice-daily rabeprazole for reflux esophagitis patients refractory to standard once-daily administration of PPI: the Japan-based TWICE study. Am J Gastroenterol. 2012;107(4):522-30.

26. Takeuchi K. Pathogenesis of NSAID-induced gastric damage: importance of cyclooxygenase inhibition and gastric hypermotility. World J Gastroenterol. 2012;18(18):2147-60.

27. Watanabe T, Higuchi K, Tominaga K, Fujiwara Y, Arakawa T. Cytoprotective effect of rabeprazole against ethanol-induced gastric mucosal damage: possible involvement of nitric oxide. Drugs Exp Clin Res. 2000;26(2):41-5.

28. Hamaguchi M, Watanabe T, Higuchi K, Tominaga K, Fujiwara Y, Arakawa T. Mechanisms and roles of neutrophil infiltration in stress-induced gastric injury in rats. Dig Dis Sci. 2001; 46(12):2708-15.

29. Arakawa T, Kobayashi K, Yoshikawa T, Tarnawski A. Rebamipide: overview of its mechanisms of action and efficacy in mucosal protection and ulcer healing. Dig Dis Sci. 1998;43(9 Suppl):5S-13S.

30. Yanaka A, Zhang S, Sato D, Tauchi M, Suzuki H, Shibahara T, et al. Geranylgeranylacetone protects the human gastric mucosa 
from diclofenac-induced injury via induction of heat shock protein 70. Digestion. 2007;75(2-3):148-55.

31. Kase Y, Yuzurihara M, Iizuka S, Ishige A, Komatsu Y. The effects of hange-shashin-to on gastric function in comparison with sho-saiko-to. Biol Pharm Bull. 1997;20(11):1155-9.

32. Goso Y, Ogata Y, Ishihara K, Hotta K. Effects of traditional herbal medicine on gastric mucin against ethanol-induced gastric injury in rats. Comp Biochem Physiol C Pharmacol Toxicol Endocrinol. 1996;113(1):17-21.

33. Arakawa T, Higuchi K, Fujiwara Y, Watanabe T, Tominaga K, Hayakawa T, et al. Gastroprotection by Liu-Jun-Zi-Tang (TJ43): possible mediation of nitric oxide but not prostaglandins or sulfhydryls. Drugs Exp Clin Res. 1999;25(5):207-10.

34. Ishii E. Genus Helicobacter pylori in stomach and antibacterial activity of natural materials against Helicobacter pylori. Gastroenterol Semin. 1997;68:143-52.

35. Higuchi K, Arakawa T, Ando K, Fujiwara Y, Uchida T, Kuroki T. Eradication of Helicobacter pylori with a Chinese herbal medicine without emergence of resistant colonies. Am J Gastroenterol. 1999;94:1419-20.

36. Hamasaki N, Ishii E, Tominaga K, Tezuka Y, Nagaoka T, Kadota S, et al. Highly selective antibacterial activity of novel alkyl quinolone alkaloids from a Chinese herbal medicine, Gosyuyu (Wu-Chu-Yu), against Helicobacter pylori in vitro. Microbiol Immunol. 2000;44(1):9-15.

37. Tominaga K, Higuchi K, Hamasaki N, Hamaguchi M, Takashima $\mathrm{T}$, Tanigawa $\mathrm{T}$, et al. In vivo action of novel alkyl methyl quinolone alkaloids against Helicobacter pylori. J Antimicrob Chemother. 2002;50(4):547-52.

38. Halder SL, Locke GR 3rd, Tally NJ, Fett SL, Zinsmeister AR, Melton LJ 3rd. Impact of functional gastrointestinal disorders on health-related quality of life: a population-based case control study. Aliment Pharmacol Ther. 2004;19:233-42.

39. Drossman DA. The functional gastrointestinal disorders and the Rome III process. Gastroenterology. 2006;130:1377-90.

40. Tominaga K, Higuchi K, Ochi M, Kadouchi K, Kawamura E, Tanigawa $\mathrm{T}$, et al. Concurrent assessment of reservoir and emptying of the stomach for dyspepsia patients. Hepatogastroenterology. 2008;55:744-9.

41. el-Omar E, Penmann I, Ardill JE, McColl KE. A substantial proportion of non-ulcer dyspepsia patients have the same abnormality of acid secretion as duodenal ulcer patients. Gut. 1995;36:534-8.

42. Salet GA, Samsom M, Roelofs JM, van Berge Henegouwen GP, Smout AJ, Akkermans LM. Responses to gastric distension in functional dyspepsia. Gut. 1998;42:823-9.

43. Holtmann G, Gschossmann J, Holtmann M, Holtmann M, Talley NJ. H. pylori and functional dyspepsia: increased serum antibodies as an independent risk factor? Dig Dis Sci. 2001;46:1550-7.

44. Tominaga K, Higuchi K, Iketani T, Ochi M, Kadouchi K, Tanigawa $\mathrm{T}$, et al. Comparison of gastrointestinal symptoms and psychological factors of functional dyspepsia to peptic ulcer or panic disorder patients. Inflammopharmacology. 2007;15:84-9.

45. Ochi M, Tominaga K, Iketani T, Kadouchi K, Tanigawa T, Shiba M, et al. Perfectionism underlying psychological background correlated with the symptoms of functional dyspepsia. J Gastroenterol. 2008;43:699-704.

46. Park DI, Rhee PL, Kim YH, Sung IK, Son HJ, Kim JJ, et al. Role of autonomic dysfunction in patients in patients with functional dyspepsia. Dig Liver Dis. 2001;33:464-71.

47. Savoye G, Bouin M, Denis P, Ducrotte P. Delayed postprandial fundic relaxation: a new abnormal finding in functional dyspepsia. Scand J Gastroenterol. 2005;40(3):354-5.

48. Desai KM, Zembowicz A, Sessa WC, Vane JR. Nitroxergic nerves mediate vagally induced relaxation in the isolated stomach of the guinea pig. Proc Natl Acad Sci USA. 1991; 88(24):11490-4.

49. Uno H, Arakawa T, Fukuda T, Higuchi K, Kobayashi K. Involvement of capsaicin-sensitive sensory nerves in gastric adaptive relaxation in isolated guinea-pig stomachs. Digestion. 1997;58(3):232-9.

50. Bredenoord AJ, Chial HJ, Camilleri M, Mullan BP, Murray JA. Gastric accommodation and emptying in evaluation of patients with upper gastrointestinal symptoms. Clin Gastroenterol Hepatol. 2003;1(4):264-72.

51. Kito Y, Suzuki H. Properties of Rikkunshi-to (TJ-43)-induced relaxation of rat gastric fundus smooth muscles. Am J Physiol Gastrointest Liver Physiol. 2010;298(5):G755-63.

52. Tatsuta M, Iishi H. Effect of treatment with Lui-Jun-Zi-Tang (TJ-43) on gastric emptying and gastrointestinal symptoms in dyspeptic patients. Aliment Pharmacol Ther. 1993;7:459-62.

53. Kusunoki H, Haruma K, Hata J, Ishii M, Kamada T, Yamashita $\mathrm{N}$, et al. Efficacy of Rikkunshito, a traditional Japanese medicine (Kampo), in treating functional dyspepsia. Intern Med. 2010;49(20):2195-202.

54. Shiratori M, Shoji T, Kanazawa M, Hongo M, Fukudo S. Effect of rikkunshito on gastric sensorimotor function under distention. Neurogastroenterol Motil. 2011;23(4):323-9.

55. Sato Y, Katagiri F, Itoh H, Takeyama M. Effects of some kampo medicines on plasma levels of neuropeptide $\mathrm{Y}$ under venipuncture stress. Biol Pharm Bull. 2005;28(9):1757-61.

56. Fujitsuka N, Asakawa A, Uezono Y, Minami K, Ymaguchi T, Niijima A, et al. Potentiation of ghrelin signaling attenuates cancer anorexia-cachexia and prolongs survival. Transl Psychiatry. 2011;26:e23.

57. Tominaga K, Higuchi K, Kadouchi K, Sasaki E, Shiba M, Watanabe T, et al. Disorder of circadian rhythm of autonomic nervous system activity and its correlation with abdominal symptoms in functional dyspepsia. Gastroenterology. 2003; 124(Supplement 1):A225.

58. Arai M, Matsumura T, Tsuchiya N, Sadakane C, Inami R, Suzuki T, et al. Rikkunshito improves the symptoms in patients with functional dyspepsia, accompanied by an increase in the level of plasma ghrelin. Hepatogastroenterology. 2012;59(113): 62-6.

59. Kawahara H, Mitani Y, Nomura M, Nose K, Yoneda A, Hasegawa T, et al. Impact of rikkunshito, an herbal medicine, on delayed gastric emptying in profoundly handicapped patients. Pediatr Surg Int. 2009;25(11):987-90.

60. Yagi M, Homma S, Kubota M, Inuma Y, Kanada S, Kinoshita $\mathrm{Y}$, et al. The herbal medicine Rikkunshi-to stimulates and coordinates the gastric myoelectric activity in post-operative dyspeptic children after gastrointestinal surgery. Pediatr Surg Int. 2004;19(12):760-5.

61. Oikawa T, Ito G, Hoshino T, Koyama H, Hanawa T. Hangekobokuto (Banxia-houpo-tang), a kampo medicine that treats functional dyspepsia. Evid Based Complement Alternat Med. 2009;6(3):375-8.

62. Nakamura T, Sakai A, Isogami I, Noda K, Ueno K, Yano S. Abatement of morphine-induced slowing in gastrointestinal transit by Dai-kenchu-to, a traditional Japanese herbal medicine. Jpn J Pharmacol. 2002;88(2):217-21.

63. Satoh K, Kase Y, Yuzurihara M, Mizoguchi K, Kurauchi K, Ishige A. Effect of Dai-kenchu-to (Da-Jian-Zhong-Tang) on the delayed intestinal propulsion induced by chlorpromazine in mice. J Ethnopharmacol. 2003;86(1):37-44.

64. Hayakawa T, Kase Y, Saito K, Hashimoto K, Ishige A, Komatsu Y, et al. Effects of Dai-kenchu-to on intestinal obstruction following laparotomy. J Smooth Muscle Res. 1999;35(2):47-54.

65. Fukuda H, Chen C, Mantyh C, Ludwig K, Pappas TN, Takahashi $\mathrm{T}$. The herbal medicine, Dai-Kenchu-to, accelerates delayed 
gastrointestinal transit after the operation in rats. J Surg Res. 2006;131(2):290-5.

66. Tokita Y, Satoh K, Sakaguchi M, Endoh Y, Mori I, Yuzurihara $\mathrm{M}$, et al. The preventive effect of Daikenchuto on postoperative adhesion-induced intestinal obstruction in rats. Inflammopharmacology. 2007;15(2):65-6.

67. Satoh K, Hayakawa T, Kase Y, Ishige A, Sasaki H, Nishikawa $\mathrm{S}$, et al. Mechanisms for contractile effect of Dai-kenchu-to in isolated guinea pig ileum. Dig Dis Sci. 2001;46(2):250-6.

68. Satoh K, Kase Y, Hayakawa T, Murata P, Ishige A, Sasaki H. Dai-kenchu-to enhances accelerated small intestinal movement. Biol Pharm Bull. 2001;24(10):1122-6.

69. Hayakawa T, Kase Y, Saito K, Hashimoto K, Ishige A, Komatsu Y, et al. Pharmacological studies of the effect of Dai-kenchu-to on spontaneous contraction of isolated rabbit jejunum. J Smooth Muscle Res. 1999;35(2):55-62.

70. Shibata C, Sasaki I, Naito H, Ueno T, Matsuno S. The herbal medicine Dai-Kenchu-Tou stimulates upper gut motility through cholinergic and 5-hydroxytryptamine 3 receptors in conscious dogs. Surgery. 1999;126(5):918-24.

71. Jin XL, Shibata C, Naito H, Ueno T, Funayama Y, Fukushima $\mathrm{K}$, et al. Intraduodenal and intrajejunal administration of the herbal medicine, dai-kenchu-tou, stimulates small intestinal motility via cholinergic receptors in conscious dogs. Dig Dis Sci. 2001;46(6):1171-6.

72. Kito Y, Suzuki H. Effects of Dai-kenchu-to on spontaneous activity in the mouse small intestine. J Smooth Muscle Res. 2006;42(6):189-201.

73. Tokita Y, Yamamoto M, Satoh K, Nishiyama M, lizuka S, Imamura $S$, et al. Possible involvement of the transient receptor potential vanilloid type 1 channel in postoperative adhesive obstruction and its prevention by a kampo (traditional Japanese) medicine, daikenchuto. J Pharmacol Sci. 2011;115(1):75-83.

74. Chikakiyo M, Shimada M, Nakao T, Higashijima J, Yoshikawa $\mathrm{K}$, Nishioka M, et al. Kampo medicine "Dai-kenchu-to" prevents CPT-11-induced small-intestinal injury in rats. Surg Today. 2012;42(1):60-7.

75. Yoshikawa K, Kurita N, Higashijima J, Miyatani T, Miyamoto $\mathrm{H}$, Nishioka M, et al. Kampo medicine "Dai-kenchu-to" prevents bacterial translocation in rats. Dig Dis Sci. 2008;53(7): 1824-31.

76. Satoh K, Hashimoto K, Hayakawa T, Ishige A, Kaneko M, Ogihara S, et al. Mechanism of atropine-resistant contraction induced by Dai-kenchu-to in guinea pig ileum. Jpn J Pharmacol. 2001;86(1):32-7.

77. Manabe N, Camilleri M, Rao A, Wong BS, Burton D, Busciglio I, et al. Effect of daikenchuto (TU-100) on gastrointestinal and colonic transit in humans. Am J Physiol Gastrointest Liver Physiol. 2010;298(6):G970-5.

78. Sato Y, Inoue S, Katagiri F, Itoh H, Takeyama M. Effects of pirenzepine on Dai-kenchu-to-induced elevation of the plasma neuropeptide levels in humans. Biol Pharm Bull. 2006;29(1): 166-71.

79. Endo S, Nishida T, Ishikawa K, Nakajima K, Hasegawa J, Kitagawa T, et al. Dai-kenchu-to, a Chinese herbal medicine, improves stasis of patients with total gastrectomy and jejunal pouch interposition. Am J Surg. 2006;192(1):9-13.

80. Itoh T, Yamakawa J, Mai M, Yamaguchi N, Kanda T. The effect of the herbal medicine dai-kenchu-to on post-operative ileus. J Int Med Res. 2002;30(4):428-32.

81. Takeda T, Kamiura S, Kimura T. Effectiveness of the herbal medicine daikenchuto for radiation-induced enteritis. J Altern Complement Med. 2008;14(6):753-5.

82. Ogasawara T, Morine Y, Ikemoto T, Imura S, Fujii M, Soejima $\mathrm{Y}$, et al. Influence of Dai-kenchu-to (DKT) on human portal blood flow. Hepatogastroenterology. 2008;55(82-83):574-7.
83. Takayama S, Seki T, Watanabe M, Monma Y, Sugita N, Konno $\mathrm{S}$, et al. The herbal medicine Daikenchuto increases blood flow in the superior mesenteric artery. Tohoku J Exp Med. 2009;219(4):319-30.

84. Murata P, Kase Y, Ishige A, Sasaki H, Kurosawa S, Nakamura T. The herbal medicine Dai-kenchu-to and one of its active components [6]-shogaol increase intestinal blood flow in rats. Life Sci. 2002;70(17):2061-70.

85. Kawasaki N, Nakada K, Nakayoshi T, Furukawa Y, Suzuki Y, Hanyu N, Yanaga K. Effect of Dai-kenchu-to on gastrointestinal motility based on differences in the site and timing of administration. Dig Dis Sci. 2007;52(10):2684-94.

86. Wood MJ, Hyman NH, Mawe GM. The effects of daikenchuto (DKT) on propulsive motility in the colon. J Surg Res. 2010;164(1):84-90.

87. Nagano T, Itoh H, Takeyama M. Effect of Dai-kenchu-to on levels of 3 brain-gut peptides (motilin, gastrin and somatostatin) in human plasma. Biol Pharm Bull. 1999;22(10):1131-3.

88. Nagano T, Itoh H, Takeyama M. Effects of Dai-kenchu-to on levels of 5-hydroxytryptamine (serotonin) and vasoactive intestinal peptides in human plasma. Biol Pharm Bull. 2000;23(3):352-3.

89. Kono T, Koseki T, Chiba S, Ebisawa Y, Chisato N, Iwamoto J, et al. Colonic vascular conductance increased by Daikenchuto via calcitonin gene-related peptide and receptor-activity modifying protein 1. J Surg Res. 2008;150(1):78-84.

90. Sato Y, Katagiri F, Inoue S, Itoh H, Takeyama M. Dai-kenchuto raises levels of calcitonin gene-related peptide and substance P in human plasma. Biol Pharm Bull. 2004;27(11):1875-7.

91. Kawahara H, Yanaga K. The herbal medicine Dai-Kenchu-To directly stimulatescolonic motility. Surg Today. 2009;39(2):175-7.

92. Kaiho T, Tanaka T, Tsuchiya S, Yanagisawa S, Takeuchi O, Miura M, et al. Effect of the herbal medicine Dai-kenchu-to for serum ammonia in hepatectomized patients. Hepatogastroenterology. 2005;52(61):161-5.

93. Suehiro T, Matsumata T, Shikada Y, Sugimachi K. The effect of the herbal medicines dai-kenchu-to and keishi-bukuryo-gan on bowel movement after colorectal surgery. Hepatogastroenterology. 2005;52(61):97-100.

94. Ohya T, Usui Y, Arii S, Iwai T, Susumu T. Effect of dai-kenchuto on obstructive bowel disease in children. Am J Chin Med. 2003;31(1):129-35.

95. Iwai N, Kume Y, Kimura O, Ono S, Aoi S, Tsuda T. Effects of herbal medicine Dai-Kenchu-to on anorectal function in children with severe constipation. Eur J Pediatr Surg. 2007; 17(2):115-8.

96. Takagi A, Yagi M, Tanaka Y, Asagiri K, Asakawa T, Tanaka H, et al. The herbal medicine daikenchuto ameliorates an impaired anorectal motor activity in postoperative pediatric patients with an anorectal malformation—a pilot study. Int Surg. 2010;95(4): $350-5$.

97. Sakakibara R, Odaka T, Lui Z, Uchiyama T, Yamaguchi K, Yamaguchi T, et al. Dietary herb extract dai-kenchu-to ameliorates constipation in parkinsonian patients (Parkinson's disease and multiple system atrophy). Mov Disord. 2005;20(2):261-2.

98. Kono T, Omiya Y, Hira Y, Kaneko A, Chiba S, Suzuki T, et al. Daikenchuto (TU-100) ameliorates colon microvascular dysfunction via endogenous adrenomedullin in Crohn's disease rat model. J Gastroenterol. 2011;46(10):1187-96.

99. Kono T, Kaneko A, Hira Y, Suzuki T, Chisato N, Ohtake N, et al. Anti-colitis and -adhesion effects of daikenchuto via endogenous adrenomedullin enhancement in Crohn's disease mouse model. J Crohns Colitis. 2010;4(2):161-70.

100. Akiho H, Nakamura K. Daikenchuto ameliorates muscle hypercontractility in a murine T-cell-mediated persistent gut motor dysfunction model. Digestion. 2011;83(3):173-9. 
101. Inoue K, Naito Y, Takagi T, Hayashi N, Hirai Y, Mizushima K, et al. Daikenchuto, a kampo medicine, regulates intestinal fibrosis associated with decreasing expression of heat shock protein 47 and collagen content in a rat colitis model. Biol Pharm Bull. 2011;34(11):1659-65.

102. Yasunaga H, Miyata H, Horiguchi H, Kuwabara K, Hashimoto $\mathrm{H}$, Matsuda S. Effect of the Japanese herbal kampo medicine dai-kenchu-to on postoperative adhesive small bowel obstruction requiring long-tube decompression: a propensity score analysis. Evid Based Complement Alternat Med. 2011;2011: 264289. doi: $10.1155 / 2011 / 264289$.

103. Yoshikawa K, Shimada M, Nishioka M, Kurita N, Iwata T, Morimoto S, et al. The effects of the Kampo medicine (Japanese herbal medicine) "Daikenchuto" on the surgical inflammatory response following laparoscopic colorectal resection. Surg Today. 2012;42(7):646-51.

104. Kase Y, Hayakawa T, Takeda S, Ishige A, Aburada M, Okada M. Pharmacological studies on antidiarrheal effects of Hangeshashin-to. Biol Pharm Bull. 1996;19(10):1367-70.

105. Kase Y, Hayakawa T, Ishige A, Aburada M, Komatsu Y. The effects of Hange-shashin-to on the content of prostaglandin E2 and water absorption in the large intestine of rats. Biol Pharm Bull. 1997;20(9):954-7.

106. Kase Y, Saitoh K, Ishige A, Komatsu Y. Mechanisms by which Hange-shashin-to reduces prostaglandin E2 levels. Biol Pharm Bull. 1998;21(12):1277-81.

107. Kase Y, Saitoh K, Yuzurihara M, Ishige A, Komatsu Y. Effects of Hange-shashin-to on cholera toxin-induced fluid secretion in the small intestine of rats. Biol Pharm Bull. 1998;21(2):117-20.

108. Kase Y, Saitoh K, Makino B, Hashimoto K, Ishige A, Komatsu Y. Relationship between the antidiarrhoeal effects of HangeShashin-To and its active components. Phytother Res. 1999;13(6):468-73.

109. Naito T, Itoh H, Yasunaga F, Takeyama M. Hange-shashin-to raises levels of somatostatin, motilin, and gastrin in the plasma of healthy subjects. Biol Pharm Bull. 2002;25(3):327-31.

110. Naito T, Itoh H, Takeyama M. Comparison of the effects of hange-shashin-to and rikkunshi-to on human plasma calcitonin gene-related peptide and substance P levels. Biol Pharm Bull. 2003;26(8):1104-7.

111. Kawashima K, Nomura A, Makino T, Saito K, Kano Y. Pharmacological properties of traditional medicine (XXIX): effect of Hange-shashin-to and the combinations of its herbal constituents on rat experimental colitis. Biol Pharm Bull. 2004;27(10):1599-603.
112. Takasuna K, Kasai Y, Kitano Y, Mori K, Kobayashi R, Hagiwara $\mathrm{T}$, et al. Protective effects of kampo medicines and baicalin against intestinal toxicity of a new anticancer camptothecin derivative, irinotecan hydrochloride (CPT-11), in Rats. Jpn J Pharmacol. 1995;86(10):978-84.

113. Takasuna K, Hagiwara T, Watanabe K, Onose S, Yoshida S, Kumazawa E, et al. Optimal antidiarrhea treatment for antitumor agent irinotecan hydrochloride (CPT-11)-induced delayed diarrhea. Cnacer Chemother Pharmacol. 2006;58:494-503.

114. Kase Y, Hayakawa T, Aburada M, Komatsu Y, Kamataki T. Preventive effects of Hange-shashin-to on irinotecan hydrochloride-caused diarrhea and its relevance to the colonic prostaglandin E2 and water absorption in the rat. Jpn J Pharmacol. 1997;75(4):407-13.

115. Mori K, Kondo T, Kamiyama Y, Kano Y, Tominaga K. Preventive effect of Kampo medicine (Hangeshashin-to) against irinotecan-induced diarrhea in advanced non-small-cell lung cancer. Cnacer Chemother Pharmacol. 2003;51:403-6.

116. Naito T, Itoh H, Yasunaga F, Takeyama M. Rikkunshi-to raises levels of somatostatin and gastrin in human plasma. Biol Pharm Bull. 2001;24(7):841-3.

117. Naito T, Itoh H, Takeyama M. Some gastrointestinal function regulatory Kampo medicines have modulatory effects on human plasma adrenocorticotropic hormone and cortisol levels with continual stress exposure. Biol Pharm Bull. 2003;26(1):101-4.

118. Yakabi K, Kurosawa S, Tamai M, Yuzurihara M, Nahata M, Ohno S, et al. Rikkunshito and 5-HT2C receptor antagonist improve cisplatin-induced anorexia via hypothalamic ghrelin interaction. Regul Pept. 2010;161(1-3):97-105.

119. Sadakane C, Muto S, Nakagawa K, Ohnishi S, Saegusa Y, Nahata M, et al. 10-Gingerol, a component of rikkunshito, improves cisplatin-induced anorexia by inhibiting acylated ghrelin degradation. Biochem Biophys Res Commun. 2011; 412(3):506-11.

120. Ohno T, Yanai M, Ando H, Toyomasu Y, Ogawa A, Morita H, et al. Rikkunshito, a traditional Japanese medicine, suppresses cisplatin-induced anorexia in humans. Clin Exp Gastroenterol. 2011;4:291-6.

121. Seike J, Sawada T, Kawakita N, Yamamoto Y, Yuasa Y, Yamai $\mathrm{H}$, et al. A new candidate supporting drug, rikkunshito, for the QOL in advanced esophageal cancer patients with chemotherapy using docetaxel/5-FU/CDDP. Int J Surg Oncol. 2011;2011: 715623. 